\title{
The Effects of Task Order Administration on Test Scores from the Trail Making Test: Near-Infrared Spectroscopy Investigations
}

\author{
Chie Takeda $^{1 *}$, Masako Notoya ${ }^{2}$, Nobuyuki Sunahara ${ }^{1}$ \\ ${ }^{1}$ Faculty of Health Sciences, Institute of Medical, Pharmaceutical and Health Sciences, Kanazawa University, Kanazawa, Japan \\ ${ }^{2}$ Department of Speech and Hearing Sciences and Disorders, Faculty of Health and Medical Sciences, Kyoto University of \\ Advanced Science, Kyoto, Japan \\ Email: ${ }^{*}$-takeda@staff.kanazawa-u.ac.jp
}

How to cite this paper: Takeda, C., Notoya, M. and Sunahara, N. (2020) The Effects of Task Order Administration on Test Scores from the Trail Making Test: Near-Infrared Spectroscopy Investigations. World Journal of Neuroscience, 10, 68-78. https://doi.org/10.4236/wjns.2020.101008

Received: January 8, 2020

Accepted: February 9, 2020

Published: February 12, 2020

Copyright $\odot 2020$ by author(s) and Scientific Research Publishing Inc. This work is licensed under the Creative Commons Attribution International License (CC BY 4.0).

http://creativecommons.org/licenses/by/4.0/

\begin{abstract}
This research examines the effects of task administration on Trail Making Test performance. The TMT consists of two parts, TMT Part A and TMT Part B. Generally, it has been believed that the order the two parts are completed does not influence test results; however, there is one previous study that has suggested that the order is indeed an influential factor on test scores. To measure frontal lobe function, the present study used near-infrared spectroscopy to measure changes in oxygenated hemoglobin (oxy- $\mathrm{Hb}$ ) in 48 young, healthy Japanese subjects as they completed Parts A and B. We subtracted the change in oxy-Hb while completing Part A from that while completing Part B (B-A Oxy-Hb) for a comparison to facilitate an investigation of how, or if, the order in which the TMT is taken influences test success. We found that when Part A was completed first, there were only small changes in the B-A oxy-Hb, but when Part B was completed first, there were larger changes. This study indicates that the order the tests are completed in may influence outcomes, thus assessments using the TMT must follow a consistent task order.
\end{abstract}

\section{Keywords}

Near-Infrared Spectroscopy, Prefrontal Cortex, Trail Making Test, Cognitive Function, Healthy Subjects

\section{Introduction}

The Trail Making Test (TMT) is a frontal lobe assessment battery that is widely 
used in clinical practice. It was developed as part of the US Army Individual Test Battery [1]. The TMT consists of two parts, TMT Part A and TMT Part B. In Part A, numbers from 1 to 25 are randomly scattered on a sheet. Subjects are asked to draw an unbroken line through them in numerical order as quickly and accurately as they can. In Part B, the instructions are the same, except there are numbers from 1 to 13 and 12 Japanese characters (letters) randomly scattered on the sheet, and the line must pass alternately through the figures and Japanese characters (letters) (e.g., 1, A, 2, B, ...). In Japan, two versions of the TMT are used in clinical settings: the version in the Halstead-Reitan Test Battery [2] and the version designed by Kashima et al. [3], which are considered the standard in Japan.

The test compares brain function in healthy people to functions in people suffering from diffuse cerebral damage, which is associated with low performance due to injuries to the frontal lobe [4]. In studies where participants performed a TMT, using functional magnetic resonance imaging (fMRI) measurements, brain activity was detected mainly in the frontal lobe [5] [6].

In near-infrared spectroscopy (NIRS) measurements, the concentrations of oxygenated hemoglobin (oxy- $\mathrm{Hb}$ ) in the frontal lobe were also recorded while subjects took the TMT Parts A and B. During Part B, higher concentrations of oxy-Hb were observed than those completing Part A [7] [8] [9] [10], suggesting that the frontal lobe is activated during the TMT.

Age, intelligence quotient (IQ), and education are believed to influence TMT performance [11] [12] [13] [14]. In a previous study of TMT performance in subjects who had no differences in age or education, three additional factors seemed to also influence brain activation: gender, knowledge of the TMT (whether a subject had prior knowledge of the task), and whether subjects performed Part A or Part B first [9].

As stated, there is conflicting research regarding whether the order the test is given in (Part A first or Part B first) affects performance outcomes. The rationale for claiming that there are differences is because Part B differs qualitatively from Part A [15]. In a previous study, we identified the order of TMT task performance as an influential factor in performance by measuring changes in oxy- $\mathrm{Hb}$ before and after task performance [9]. However, we did not compare performance on Part A and B directly. If the order does affect performance, it may also influence the interpretation of the results. Thus, in the present study, we examined how the order of TMT test administration influences TMT performance in detail.

\section{Methods}

\subsection{Subjects}

The subjects were 48 healthy, right-handed Japanese adults who ranged in age from 20 to 29 years old. There were 24 males and 24 females, with a mean age of $22.8 \pm 2.5$ years, and the mean years of education years of $16.0 \pm 1.2$ years. They 
signed informed consent forms after the procedures were explained. Their visual acuity was normal, and none had any medical history, such as neurological disorders, that would have influenced the data collected in this study. The protocol for this study was approved by the Ethics Review Board of the Medical Research Division, Kanazawa University (No. 252).

\subsection{NIRS Measurements}

The ETG-4000 near-infrared Spectroscopy System (NIRS) (Hitachi Medical Corporation, Tokyo, Japan) uses two wavelengths of near-infrared light, $695 \mathrm{~nm}$ and $830 \mathrm{~nm}$. The ETG-4000 was used to measure areas of the brain in both hemispheres. Fifteen probes were placed in each hemisphere (8 emission and 7 detection probes) (Figure 1). It was possible to obtain measurements from 22 channels in each hemisphere, yielding measurements from 44 channels. Of these channels, data from 24 gray channels were used to monitor prefrontal cortex activity (Figure 2). To unify the measurement sites, the lowest row of emission and detection probes was placed on a line connecting T3 with T4, which was described in the international 10 - 20 electrode system used for electroencephalography.

\subsection{TMT}

In this study, we used Kashima's version of the TMT (Figure 3). In both Parts A and $\mathrm{B}$, we created two patterns of TMT with a new arrangement, which referred to Kashima's TMT in consideration of the learning effect. In addition to TMT of Kashima, each part used 3 patterns of TMT as a subject. A pilot test found no difference in the subjective degree of difficulty or performance time among these tests. The subjects were divided randomly into two groups initially assigned to perform either TMT Part A or B (Group 1 and Group 2, respectively). Both groups

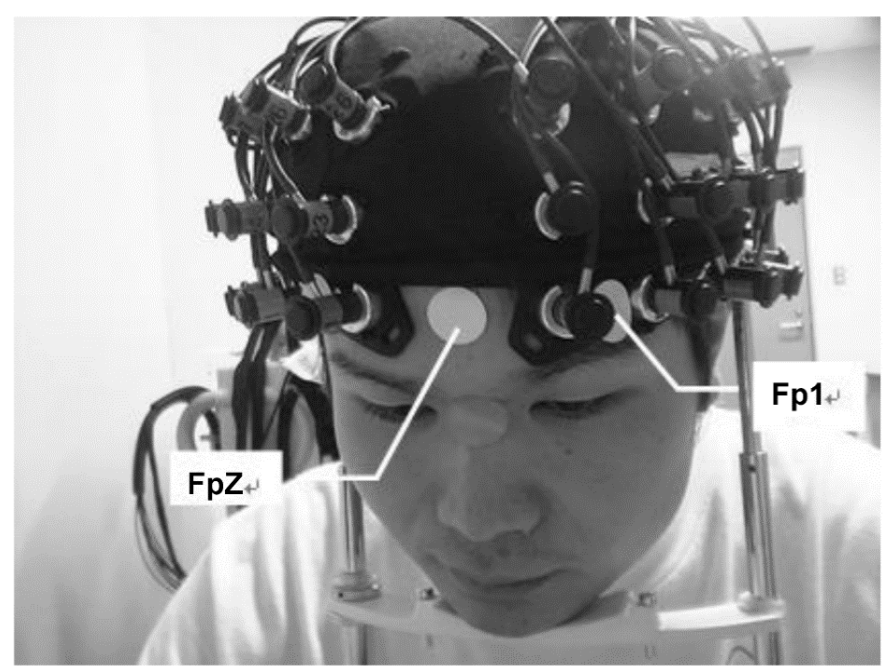

Figure 1. Positions of the NIRS probes. To unify the measurement sites, the lowest row of emission and detection probes were placed on a line connecting T3 with T4, which are described in the international 10 - 20 electrode system used for electroencephalography. 


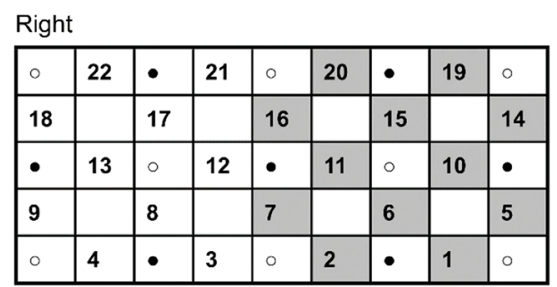

Left
\begin{tabular}{|l|l|l|l|l|l|l|l|l|}
\hline$\circ$ & 22 & $\bullet$ & 21 & $\circ$ & 20 & $\bullet$ & 19 & $\circ$ \\
\hline 18 & & 17 & & 16 & & 15 & & 14 \\
\hline$\bullet$ & 13 & $\circ$ & 12 & $\bullet$ & 11 & $\circ$ & 10 & $\bullet$ \\
\hline 9 & & 8 & & 7 & & 6 & & 5 \\
\hline$\circ$ & 4 & $\bullet$ & 3 & $\circ$ & 2 & $\bullet$ & 1 & $\circ$ \\
\hline
\end{tabular}

Figure 2. Measurement channels. In this, the gray part represents the frontal lobe; $\bigcirc$ represents emission probes; and represents detection probes.
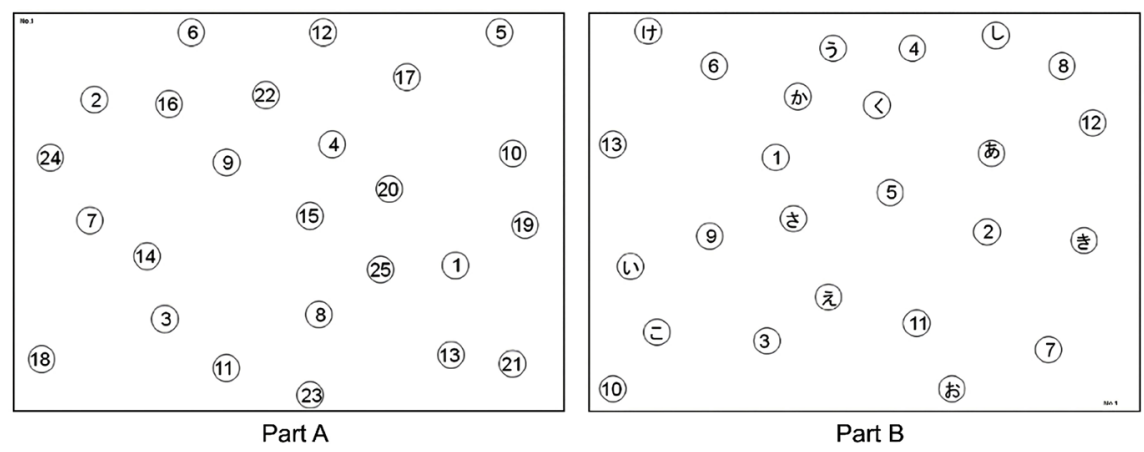

Figure 3. Trail making test.

had the same number of participants. A distinction was also made between participants who were not familiar with the TMT (Uninformed group) and anyone who had previously taken it (Informed group). The number of participants in the Uninformed and Informed sub-groups in Groups 1 and 2 was equal. The male-to-female ratio was 1:1 for all groups. There is no difference in education level among all groups.

\subsection{Research Design}

Participants were instructed to perform the TMT with a pencil sitting at a desk in a quiet room, just as the test is given in clinical practice. Because a previous study reported that (head movement), cephalic motion-related changes in cerebral blood flow [16] had a bearing on TMT outcomes, so in the instant study, cephalic motion was restricted using a jaw rest. During the rest periods, the subjects were also instructed to gaze at a green point on the desk without moving their heads. They were also given three-minute rest periods between sessions during which they were fitted with neural probes.

The organization of the measurement session was as follows: Session 1 was comprised of a 10 -second pre-scan, followed by alternating four 60 -second rest periods with three 30 -second performance periods.

During the performance periods, the subjects performed the task three times in one session. Sheets of paper were placed in front of the subjects immediately before each task began. The subjects started the TMT on a pre-agreed verbal cue, "please start". After 30 seconds, they were asked to, "please stop". Then the proctor collected the test sheets.

Typically, when administering the TMT, the interval required to complete the 
tasks is recorded. However, in this study, the number of tasks that were accomplished within 30 seconds was recorded because the NIRS was used for measurements. A previous study showed that the number of Part B tasks completed within 30 seconds was significantly smaller than the number of Part A tasks [8]. This was consistent with the finding that Part B took longer for participants to complete [5]. Therefore, a similar method was employed in this study. The number tasks completed in 30 seconds was designated as the TMT score.

\subsection{NIRS Data Analysis}

Any relative changes in concentrations of oxy-Hb were measured. Before the start of a session, data was collect for ten seconds on the rates of change in oxy-Hb levels. Data was collected during the 30 seconds of the test and for the 50 seconds after three rounds of tests completion; then collected data was averaged. Subsequently, we conducted a zero-point correction so that the mean rate of change for the ten seconds before the start of the test was set to zero. To calculate the differences between the oxy-Hb levels for Parts A and B, the oxy-Hb level following completion of Part A was subtracted from the oxy-Hb level following completion of Part B. Subsequently, the mean rate of change in the oxy-Hb level for the 30 seconds of test activity was calculated for each channel (B-A) (Figure 4).

\subsection{Statistical Analysis}

The mean TMT scores for the groups were compared in order of TMT test order using an unpaired Student's t-test, with $\mathrm{p}=0.05$ set as the significance threshold. In both TMT test order groups, the mean changes in the oxy-Hb level of $\mathrm{B}-\mathrm{A}$ for each channel were calculated for the t-test with a null hypothesis of $\mathrm{H} 0: \mu=0$. Multiple regression analyses were performed to investigate oxy-Hb changes (B-A) using the order the TMT was performed (order), prior knowledge of the TMT (knowledge), and gender as independent variables. Statistical analysis was performed using JMP ${ }^{\infty} 10$ statistical measuring software (SAS Institute Inc., Cary, NC, USA).

\section{Results}

\subsection{TMT Scores}

TMT score indicates the number of TMT targets that can be reached in 30 seconds [8] [9]. To measure changes in B-A oxy-Hb levels, multiple regression analyses were performed on the three factors as independent variables: gender, prior knowledge of the TMT, and the order in which the parts of the TMT were administered.

The mean Part A scores were 17.0 \pm 3.0 in Group 1 and $17.0 \pm 2.9$ in Group 2, showing no significant difference between the group scores $(\mathrm{t}=-0.034, \mathrm{p}=$ 0.974). The mean Part B scores were $14.5 \pm 2.8$ in Group 1 and $12.6 \pm 2.7$ in Group 2, demonstrating a significant difference $(\mathrm{t}=-2.424, \mathrm{p}=0.024)$. 


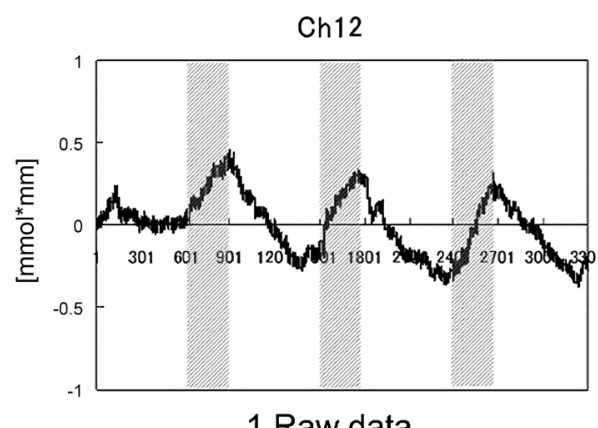

1. Raw data

Ch12

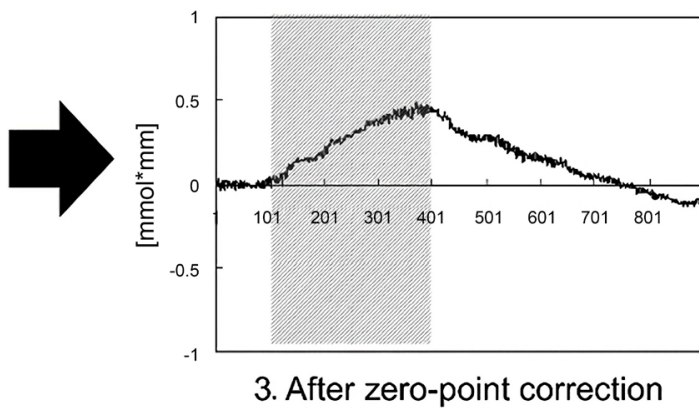

Ch12

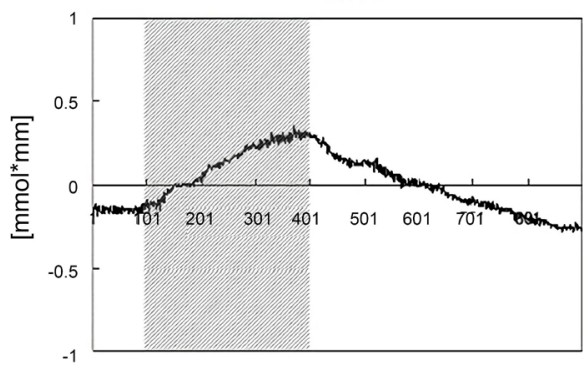

2. Average

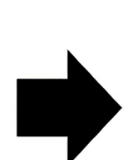

4. Calculating the mean rate of change in the oxy- $\mathrm{Hb}$ level for 30 seconds of the test $=($ Part B - Part A $) / 30$ s

Figure 4. Processing method for the NIRS data. Average $=$ (the rates of change in the oxy-Hb level for 10 seconds before the start of the test, 30 seconds during the test, and 50 seconds after its completion for three times)/3. After zero-point correction $=$ (average) $-($ the mean rate of change in the oxy-Hb level for 10 seconds before the start of the test); the gray hatched areas represent performance periods.

Table 1. Results according to the factors from changing B-A oxy-Hb levels in Left Ch.

\begin{tabular}{ccccc}
\hline Ch & factor & $\mathrm{R}^{2}$ & $\beta$ & P value \\
\hline 1 & Order & 0.124 & -0.337 & 0.021 \\
2 & Order & 0.107 & -0.316 & 0.032 \\
$\underline{3}$ & Order & 0.173 & -0.401 & 0.005 \\
$\underline{4}$ & Order & 0.32 & -0.54 & $<0.001$ \\
5 & Order & 0.2 & -0.413 & 0.004 \\
$\underline{7}$ & Order & 0.096 & -0.307 & 0.038 \\
$\underline{8}$ & Order & 0.248 & -0.467 & 0.001 \\
$\underline{9}$ & Order & 0.242 & -0.45 & 0.001 \\
10 & Order & 0.57 & -0.375 & 0.01 \\
11 & Order & 0.112 & -0.289 & 0.048 \\
$\underline{12}$ & Order & 0.22 & -0.423 & 0.003 \\
$\underline{13}$ & Order & 0.208 & -0.4 & 0.005 \\
14 & Order & 0.169 & -0.354 & 0.017 \\
15 & Order & 0.142 & -0.356 & 0.014 \\
$\underline{18}$ & Order & 0.218 & -0.424 & 0.003 \\
\hline
\end{tabular}

Multiple regression analyses were performed to investigate oxy-Hb changes (B-A) using the order the TMT was performed (order), prior knowledge of the TMT (knowledge), and gender as independent variables. Multiple regression analysis was performed on all channels $(n=48)$. In the table, only the channels with significant differences are shown. Independent variables selected by multiple regression analysis (factor), contribution ratio $\left(\mathrm{R}^{2}\right)$, standard $\beta(\beta)$ and $\mathrm{p}$ values are shown for each channel. The table shows channels where significant differences were found. Underlined channels correspond to the prefrontal cortex. $\mathrm{Ch}=$ channel; order $=$ the order the TMT was performed. 


\subsection{Changes in B-A Oxy-Hb Levels by Channels}

Group 1 showed no channels that increased in oxy-Hb levels. In Group 2, significant increases were detected in Channels $1,2,3,4,5,7,8,9,10,12,13$, and 18 on the left side of the brain and in Channels $1,2,3,4,5,6,7,8,9,10,11,12$, $13,15,16,17,18,19,20$, and 22 on the right side of the brain. In contrast, significant decreases in B-A oxy-Hb levels were detected in Channels 11, 15, 18, and 19 on the left side and in Channels 1, 6, and 11 on the right side in Group 1 participants; however, in Group 2, no channels recorded decreased oxy-Hb levels in any of the participants. These results indicate that brain activation was higher during Part A than during Part B in Group 1 and higher during Part B than during Part A in Group 2.

\subsection{Changes in B-A 0xy-Hb Levels for Additional Three Factors}

Multiple regression analysis was performed on all channels. Most independent variables selected as factors affecting oxy-Hb change were in the order of the TMT.

For the order in which the tasks of the TMT were administered, significant changes were detected in Channels 1, 2, 3, 4, 5, 7, 8, 9, 10,11, 12, 13, 14, 15 and 18 on the left side (Table 1) and in Channels $1,2,3,4,5,6,7,8,9,10,11,12,13$, $15,16,17,18,20,21$ and 22 on the right side of the brains of participants (Table 2). Channels corresponding to the prefrontal cortex were identified as Channels $3,4,5,7,8,9,12,13$ and 18 on the left side and Channels $1,2,5,6,7,10,11,15$, 16 and 20 on the right side. The oxy-Hb level was significantly higher in Group 2 than in Group 1.

There were significant knowledge of the TMT influences on oxy-Hb changes in Channel 9 on the right side (Table 2), and oxy-Hb levels were significantly higher in uninformed group than in informed group. Prior gender had no influence on oxy-Hb changes.

Table 2. Results according to the factors from changing B-A oxy-Hb levels in Right Ch.

\begin{tabular}{|c|c|c|c|c|}
\hline $\mathrm{Ch}$ & factor & $\mathrm{R}^{2}$ & $\beta$ & $P$ value \\
\hline$\underline{1}$ & Order & 0.307 & -0.552 & $<0.001$ \\
\hline$\underline{2}$ & Order & 0.17 & -0.412 & 0.004 \\
\hline 3 & Order & 0.139 & -0.362 & 0.013 \\
\hline 4 & Order & 0.133 & -0.335 & 0.021 \\
\hline$\underline{5}$ & Order & 0.268 & -0.5 & $<0.001$ \\
\hline$\underline{6}$ & Order & 0.232 & -0.48 & 0.001 \\
\hline$\underline{7}$ & Order & 0.154 & -0.392 & 0.007 \\
\hline 8 & Order & 0.105 & -0.32 & 0.303 \\
\hline 9 & $\begin{array}{c}\text { Order } \\
\text { knowledge }\end{array}$ & 0.226 & $\begin{array}{c}-0.364 \\
0.304\end{array}$ & $\begin{array}{l}0.009 \\
0.027\end{array}$ \\
\hline$\underline{10}$ & Order & 0.184 & -0.418 & 0.004 \\
\hline
\end{tabular}




\section{Continued}

\begin{tabular}{lllll}
\hline$\underline{11}$ & Order & 0.309 & -0.529 & $<0.001$ \\
12 & Order & 0.157 & -0.39 & 0.007 \\
13 & Order & 0.196 & -0.437 & 0.002 \\
$\underline{15}$ & Order & 0.235 & -0406 & 0.003 \\
$\underline{16}$ & Order & 0.171 & -0.398 & 0.006 \\
17 & Order & 0.171 & -0.408 & 0.005 \\
18 & Order & 0.161 & -0.375 & 0.009 \\
$\underline{20}$ & Order & 0.119 & -0.327 & 0.027 \\
21 & Order & 0.127 & -0.327 & 0.025 \\
22 & Order & 0.138 & -0.367 & 0.014 \\
\hline
\end{tabular}

Multiple regression analyses were performed to investigate oxy-Hb changes (B-A) using the order the TMT was performed (order), prior knowledge of the TMT (knowledge), and gender as independent variables. Multiple regression analysis was performed on all channels $(n=48)$. In the table, only the channels with significant differences are shown. Independent variables selected by multiple regression analysis (factor), contribution ratio $\left(\mathrm{R}^{2}\right)$, standard $\beta(\beta)$ and $\mathrm{p}$ values are shown for each channel. The table shows channels where significant differences were found. Underlined channels correspond to the prefrontal cortex. $\mathrm{Ch}=$ channel; order $=$ the order the TMT was performed; knowledge $=$ prior knowledge of the TMT.

\section{Discussion}

The order of administration of the parts of the TMT affected the oxy-Hb levels of B-A in various NIRS measurement channels. When the brain familiarizes itself with a task, cerebral blood flow decreases in the prefrontal cortex and supplementary motor area [17]. After a task is learned, cerebral blood flow decreases in the region of interest whenever the task is performed again [18] [19]. Thus, there is some consensus that brain activity decreases in the affected area after learning a task. In Group 1, participants performed Part A, a relatively easy task, first. This facilitated learning how to efficiently search for targets and connect them in order. Then they performed Part B, a more difficult task. But because of their previous experience with Part A, Group 1 was able to perform Part B smoothly. Thus, we conclude that oxy-Hb levels in the prefrontal cortex were higher in Part A than in Part B because of the previous learning experience; therefore, the TMT Part B score was higher in Group 1 than Group 2. In contrast, Group 2 performed Part B first, which made it harder for them to learn the strategy to successfully complete the TMT under the applied test condition of time constraint/activities completed used for scoring. As a result, B-A oxy-Hb levels were higher in Group 2 than in Group 1 as they did not have the benefit of learning one part before having to complete all parts of the TMT. Furthermore, brain activation occurred during the whole measurement range in Group 2, but not in Group 1. These results are consistent with the findings from a study of a word fluency task, where oxy-Hb increases in subjects with low performance tended to be higher than for subjects with a high performance level, perhaps because the former solved problems less efficiently and thus exerted greater effort. As a result, their cerebral blood volume was higher [20]. In this study, we con- 
clude that effort in task performance causes widespread brain activation.

The findings of the instant study contradict the assertion of Toyokura et al., [15] that the performance order of Part A and Part B does not affect test results. The instant study finds that following a consistent task order when using the TMT in research is likely an important factor in producing scientific results that can be compared with confidence across disciplines. TMT Part A and B are useful tests that can evaluate frontal lobe function. Therefore, it is important to follow the order of TMT in interpreting the results.

In the instant study, previous knowledge of the TMT was found in only one channel for oxy-Hb levels; Channel 9 in right area. This is consistent with our previous study [9]. However, since it is a site other than the frontal area and only $1 \mathrm{Ch}$, the experience may not have much effect. Gender did not affect the levels of B-A oxy-Hb. This suggests that the analyses of B-A oxy-Hb are not affected by gender. Previous studies have produced mixed results regarding gender difference in TMT performance. Some have produced indications that gender has an effect on performance, while others reported no gender effect [8] [13] [21] [22] [23]. Therefore, no conclusive gender effect was identified.

NIRS can detect changes in blood volume in the cerebral cortex with high temporal resolution using near-infrared light of two different wavelengths. It is non-invasive to the human body, operates quietly, and can be measured in a sitting position. Since the attention function is required like TMT, NIRS measurement is useful for tests that are recommended to be performed in a chair sitting in a quiet environment. NIRS is a tool that can be safely and easily measured when examining brain activity during task performance that cannot be determined by task performance alone.

\section{Conflicts of Interest}

In this research, there are no grants and no conflict of interest.

\section{References}

[1] Army Individual Test Battery (1944) Manual of Directions and Scoring. War Department, Adjutant General's Office, Washington DC.

[2] Reitan, R.M. and Wolfson, D. (1985) The Halstead-Reitan Neuropsychological Test Battery.

[3] Kashima, H., Handa, T., Katoh, M., Honda, T., Sakuma, K., Muramatsu, T., et al. (1986) Disorders of Attention Due to Frontal Lobe Lesion. Advances in Neurological Sciences, 30, 847-858. (In Japanese)

[4] Stuss, D.T., Bisschop, S.M., Alexander, M.P., Levine, B., Katz, D. and Izukawa, D. (2001) The Trail Making Test: A Study in Focal Lesion Patients. Psychological Assessment, 13, 230-239. https://doi.org/10.1037/1040-3590.13.2.230

[5] Moll, J., Oliveira-Souza, R., Moll, F.T., Bramati, I.E. and Andreiuolo, P.A. (2002) The Cerebral Correlates of Set-Shifting: An fMRI Study of the Trail Making Test. Arquivos de Neuro-Psiquiatria, 60, 900-905. https://doi.org/10.1590/S0004-282X2002000600002

[6] Zakzanis, K.K., Mraz, R. and Graham, S.J. (2005) An fMRI Study of the Trail Mak- 
ing Test. Neuropsychologia, 43, 1878-1886.

[7] Shibuya-Tayoshi, S., Sumitani, S., Kikuchi, K., Tanaka, T., Tayoshi, S., Ueno, S., et al. (2007) Activation of the Prefrontal Cortex during the Trail-Making Test Detected with Multichannel Near-Infrared Spectroscopy. Psychiatry and Clinical Neurosciences, 61, 616-621. https://doi.org/10.1111/j.1440-1819.2007.01727.x

[8] Shoji, C., Notoya, M. and Inoue, K. (2009) Activity of the Frontal Lobe on Near-Infrared Spectroscopy (NIRS) during Trail Making Test Performance. Journal of the Tsuruma Health Science Society, 33, 41-48. (In Japanese)

[9] Takeda, C., Notoya, M., Sunahara, N. and Inoue, K. (2011) Identification of Three Factors Influencing Trail Making Test Performance Using Multichannel Near-Infrared Spectroscopy. The Tohoku Journal of Experimental Medicine, 223, 103-112. https://doi.org/10.1620/tjem.223.103

[10] Weber, P., Lutschg, J. and Fahnenstich, H. (2005) Cerebral Hemodynamic Changes in Response to an Executive Function Task in Children with Attention-Deficit Hyperactivity Disorder Measured by Near-Infrared Spectroscopy. Journal of Developmental \& Behavioral Pediatrics, 26, 105-111. https://doi.org/10.1097/00004703-200504000-00005

[11] Abe, M., Suzuki, K., Okada, K., Miura, R., Fujii, T., Mori, E., et al. (2004) Normative Data on Tests for Frontal Lobe Functions: Trail Making Test, Verbal Fluency, Wisconsin Card Sorting Test (Keio Version). No to Shinkei, 57, 567-574. (In Japanese)

[12] Boll, T.J. and Reitan, R.M. (1973) Effect of Age on Performance of the Trail Making Test. Perceptual and Motor Skills, 36, 691-694. https://doi.org/10.2466/pms.1973.36.3.691

[13] Bornstein, R.A. (1985) Normative Data on Selected Neuropsychological Measures from a Nonclinical Sample. Journal of Clinical Psychology, 41, 651-659. https://doi.org/10.1002/1097-4679(198509)41:5<651::AID-JCLP2270410511>3.0.CO $\underline{; 2-\mathrm{C}}$

[14] Davies, A.D. (1968) The Influence of Age on Trail Making Test Performance. Journal of Clinical Psychology, 24, 96-98. https://doi.org/10.1002/1097-4679(196801)24:1<96::AID-JCLP2270240131>3.0.CO; $\underline{2-\mathrm{U}}$

[15] Toyokura, M., Tanaka, H., Furukawa, T., Yamanouchi, Y. and Murakami, K. (1996) Normal Aging Effect on Cognitive Task Performance of Information-Processing Speed: Analysis of Paced Auditory Serial Addition Task and Trail Making Test. Brain Science and Mental Disorders, 7, 401-409. (In Japanese)

[16] Kurihara, K., Kikukawa, A. and Kobayashi, A. (2003) Cerebral Oxygenation Monitor during Head-Up and -Down Tilt Using Near-Infrared Spatially Resolved Spectroscopy. Clinical Physiology and Functional Imaging, 23, 177-181. https://doi.org/10.1046/j.1475-097X.2003.00488.x

[17] Sakai, K., Hikosaka, O., Miyauchi, S., Takino, R., Sasaki, Y. and Putz, B. (1998) Transition of Brain Activation from Frontal to Parietal Areas in Visuomotor Sequence Learning. Journal of Neuroscience, 18, 1827-1840. https://doi.org/10.1523/JNEUROSCI.18-05-01827.1998

[18] Lee, H., Voss, M.W., Prakash, R.S., Boot, W.R., Vo, L.T., Basak, C., et al. (2012) Videogame Training Strategy-Induced Change in Brain Function during a Complex Visuomotor Task. Behavioural Brain Research, 232, 348-357. https://doi.org/10.1016/j.bbr.2012.03.043

[19] Ohuchida, K., Kenmotsu, H., Yamamoto, A., Sawada, K., Hayami, T., Morooka, K., et al. (2009) The Frontal Cortex Is Activated during Learning of Endoscopic Proce- 
dures. Surgical Endoscopy, 23, 2296-2301.

https://doi.org/10.1007/s00464-008-0316-Z

[20] Kameyama, M., Fukuda, M., Uehara, T. and Mikuni, M. (2004) Sex and Age Dependencies of Cerebral Blood Volume Changes during Cognitive Activation: A Multichannel Near-Infrared Spectroscopy Study. Neuroimage, 22, 1715-1721.

https://doi.org/10.1016/j.neuroimage.2004.03.050

[21] Gaudino, E.A., Geisler, M.W. and Squires, N.K. (1995) Construct Validity in the Trail Making Test: What Makes Part B Harder? Journal of Clinical and Experimental Neuropsychology, 17, 529-535. https://doi.org/10.1080/01688639508405143

[22] Waldmann, B.W., Dickson, A.L., Monahan, M.C. and Kazelskis, R. (1992) The Relationship between Intellectual Ability and Adult Performance on the Trail Making Test and the Symbol Digit Modalities Test. Journal of Clinical Psychology, 48, 360-363.

https://doi.org/10.1002/1097-4679(199205)48:3<360::AID-JCLP2270480314>3.0.CO ;2-P

[23] Wiederholt, W.C., Cahn, D., Butters, N.M., Salmon, D.P., Kritz-Silvestein, D. and Barrett-Conner, E. (1993) Effects of Age, Gender and Education on Selected Neuropsychological Tests in an Elderly Community Cohort. Journal of the American Geriatrics Society, 41, 639-647. https://doi.org/10.1111/j.1532-5415.1993.tb06738.x 